\title{
Metodología para la inclusión de criterios ambientales en las compras públicas: Caso de estudio Instituto Tecnológico de Costa Rica ${ }^{1}$
}

\section{Methodology for the inclusion of environmental criteria in public procurement: Case study Instituto Tecnológico de Costa Rica}

Juan José Gamboa-Castro², Teresa Salazar-Rojas³

Fecha de recepción: 10 de enero de 2020

Fecha de aprobación: 23 de abril de 2020

Gamboa-Castro, J.J; Salazar-Rojas, T. Metodología para la inclusión de criterios ambientales en las compras públicas: caso de estudio Instituto Tecnológico de Costa Rica. Tecnología en Marcha. Vol. 34-2. Abril-Junio 2021. Pág 60-75.

doi) https://doi.org/10.18845/tm.v34i2.4897

1 Artículo basado en el Proyecto Final de Graduación "Metodología para la inclusión de criterios ambientales en las compras públicas: Caso de estudio Instituto Tecnológico de Costa Rica" del primer autor. Disponible en http://hdl.handle.net/2238/7371

2 Ingeniero Ambiental. Ernst \& Young S.A. Costa Rica. Correo electrónico: juan.gamboa.castro@cr.ey.com. (D) https://orcid.org/0000-0003-3817-5757

3 Master en Gestión Ambiental Escuela de Química (Carrera de Ingeniería Ambiental), Instituto Tecnológico de Costa Rica. Costa Rica. Correo electrónico: tsalazar@tec.ac.cr. 


\title{
Palabras clave
}

Compras públicas verdes; contratación pública ecológica; criterios ambientales; compras públicas sostenibles; gobierno; desarrollo sostenible.

\section{Resumen}

En Costa Rica el principal consumidor de bienes y servicios es el sector público, esto le confiere la capacidad de accionar los mercados hacia modelos de producción sostenibles, lo cual ha venido intentando hacer mediante iniciativas para promover las compras verdes y sostenibles en sus instituciones estatales, sin embargo, aún existe desconocimiento y falta de experiencia en la implementación de programas de este tipo de compras. Esta investigación propone una metodología para la inclusión de criterios ambientales en los procesos de contratación administrativa, desarrollada en el Tecnológico de Costa Rica (TEC). Para esto, se priorizaron categorías de productos, se definieron criterios ambientales con sus respectivas evidencias de verificación y se realizaron entrevistas a proveedores con el fin de determinar la factibilidad de cumplimiento; por último, se clasificaron los criterios según la legislación vigente. Los grupos de productos seleccionados considerando relación de gasto - presupuesto anual de la institución, volumen de compra, cobertura interna de uso e impacto ambiental fueron mobiliario, suministros de oficina, vestuario y servicios de alimentación; resultando con mayor tasa de cumplimiento de las especificaciones, los proveedores de las dos primeras categorías. En el caso de textiles se detectó una carencia de información referente a los criterios ambientales, y para los servicios de alimentación, se evidenció la falta de capacitación de las empresas y el insuficiente avance de programas de eco etiquetado en productos cárnicos a nivel nacional.

\section{Keywords}

Green public procurement; environmental criteria; sustainable public procurement; government; sustainable development.

\begin{abstract}
The public sector is the main consumer of the country; therefore, it has the capacity to drive markets towards sustainable production models. Costa Rica has initiatives to promote green and sustainable public procurement. However, there is still lack of knowledge and lack of experience. This research proposes a methodology applicable to any public entity for the inclusion of environmental criteria in its procurement processes and was developed at Tecnológico de Costa Rica (TEC). To this end, four product categories were prioritized, environmental criteria were determined with their respective evidences of verification and market consultations were carried out to determine the feasibility of compliance. Finally, the criteria were classified according to the current legislation. The selected product groups, considering the annual expenditure of the institution-budget, purchasing volume, internal use coverage and environmental impact, were furniture, office supplies, clothing and food services; resulted in a higher compliance rate of specifications for the suppliers of the first two categories. In the case of textiles was found deficiencies about the environmental traceability of raw materials and production processes, for the latter category shows the lack of training of the companies and the insufficient progress of eco-labeling programs in meat products at national level.
\end{abstract}




\section{Introducción}

Como parte de la Agenda 2030 para el Desarrollo Sostenible, en el 2015, la ONU establece los Objetivos de Desarrollo Sostenible. Particularmente el objetivo doce corresponde a garantizar modalidades de consumo y producción sostenibles, una de las metas propuestas para este objetivo es "promover prácticas de adquisición pública que sean sostenibles, de conformidad con las políticas y prioridades nacionales" [1]

El concepto de Compras Públicas Verdes (CPV) alude exclusivamente al eje ambiental de las Compras Públicas Sostenibles (CPS), también se le conoce como Contratación Pública Ecológica (CPE) y es el campo de acción de esta investigación; de acuerdo con la Comisión de las Comunidades Europeas [2] las CPV se definen como sigue:

Proceso por el cual las autoridades públicas tratan de adquirir mercancías, servicios y obras con un impacto medioambiental reducido durante su ciclo de vida, en comparación con el de mercancías, servicios y obras con la misma función primaria que se adquirirían en su lugar.

Analizando los términos de CPS y CPV, no es posible utilizarlos indistintamente, si bien se encuentran íntimamente relacionados, la CPV tiene una óptica meramente ambiental, de acuerdo con Brammer \& Walker [3] este concepto dio origen al de CPS, pues las investigaciones relativas a los aspectos de sostenibilidad en las compras indican que el primer enfoque implementado fue el de gestión de los problemas ambientales en las cadenas de suministro.

Tanto las CPS como las CPS representan un desafío para los funcionarios dedicados a los procesos de contratación administrativa, les exige maximizar el valor por el dinero a lo largo del ciclo de vida del bien y no únicamente en el momento de la compra [4], este principio implica un cambio en el paradigma bajo el cual se adjudican los contratos comúnmente, es decir, aquel donde la oferta con el precio más bajo resulta favorecida, ya que en muchas ocasiones esta práctica resulta en la adquisición de bienes con huellas ecológicas elevadas y a largo plazo representan pérdidas para el dinero público, así como perjuicios al ambiente [5].

Al hablar de CPV un tema relevante es la definición de los criterios ambientales, ya que éstos hacen referencia a aquella característica del bien solicitado que disminuye su impacto ambiental en relación con otro cuya función es equivalente [6]. Estos criterios pueden ser de selección positiva, cuando favorecen determinados bienes con características ambientalmente amigables (por ejemplo, recipientes para alimentos compostables en un lapso no mayor a 180 días) o criterios de selección negativa, donde se engloban aquellos cuyo objetivo es excluir o restringir la participación de productos o servicios con propiedades nocivas para el ambiente (por ejemplo, telas que contengan más de 1,0 ppm de plomo dentro de su composición) [7].

Las entidades públicas deben definir e implementar mecanismos y evidencias de verificación para controlar que los criterios ambientales solicitados sean satisfechos, así como lo hacen, por ejemplo, con especificaciones de calidad, esto con el fin de asegurarse que los proveedores cuentan con pruebas técnicas para asegurar la veracidad de las características que hacen al producto menos perjudicial para el ambiente. Así, además de las especificaciones técnicas ambientales, es necesario establecer en los pliegos de condiciones de las licitaciones las disposiciones para su comprobación [8].

Las CPV son un eje transversal a considerar en el marco de ambientalización del sector público costarricense, que pretende la mejora continua en las organizaciones estatales [9]. Sin embargo, a pesar de esfuerzos realizados estudios como el de Fundación Centro de Gestión Tecnológica e Informática Industrial (CEGESTI) [10], demuestran que el sector público aún se encuentra en proceso de adaptación y requiere capacitación y herramientas para la implementación de un programa de compras verdes exitoso. La principal problemática radica en la falta de criterio 
técnico para integrar especificaciones ambientales dentro de los términos de referencia de las compras, así como la comprobación de su cumplimiento con evidencias objetivamente verificables en el contexto costarricense.

Por tanto, con el objeto de solventar tales necesidades, esta investigación plantea una metodología para la inclusión de criterios ambientales en las compras públicas, estudiando el caso del Tecnológico de Costa Rica (TEC), para lo cual se realizaron consultas a distintos proveedores, con el fin de conocer el nivel de exigencia de los criterios a solicitar en futuras contrataciones.

\section{Metodología}

\section{Definición del caso de estudio}

La investigación se desarrolló en el Tecnológico de Costa Rica (TEC), sede Central; localizado en la provincia de Cartago, cantón Cartago, distritos Oriental y Dulce Nombre. La ubicación se presenta en la figura 1.

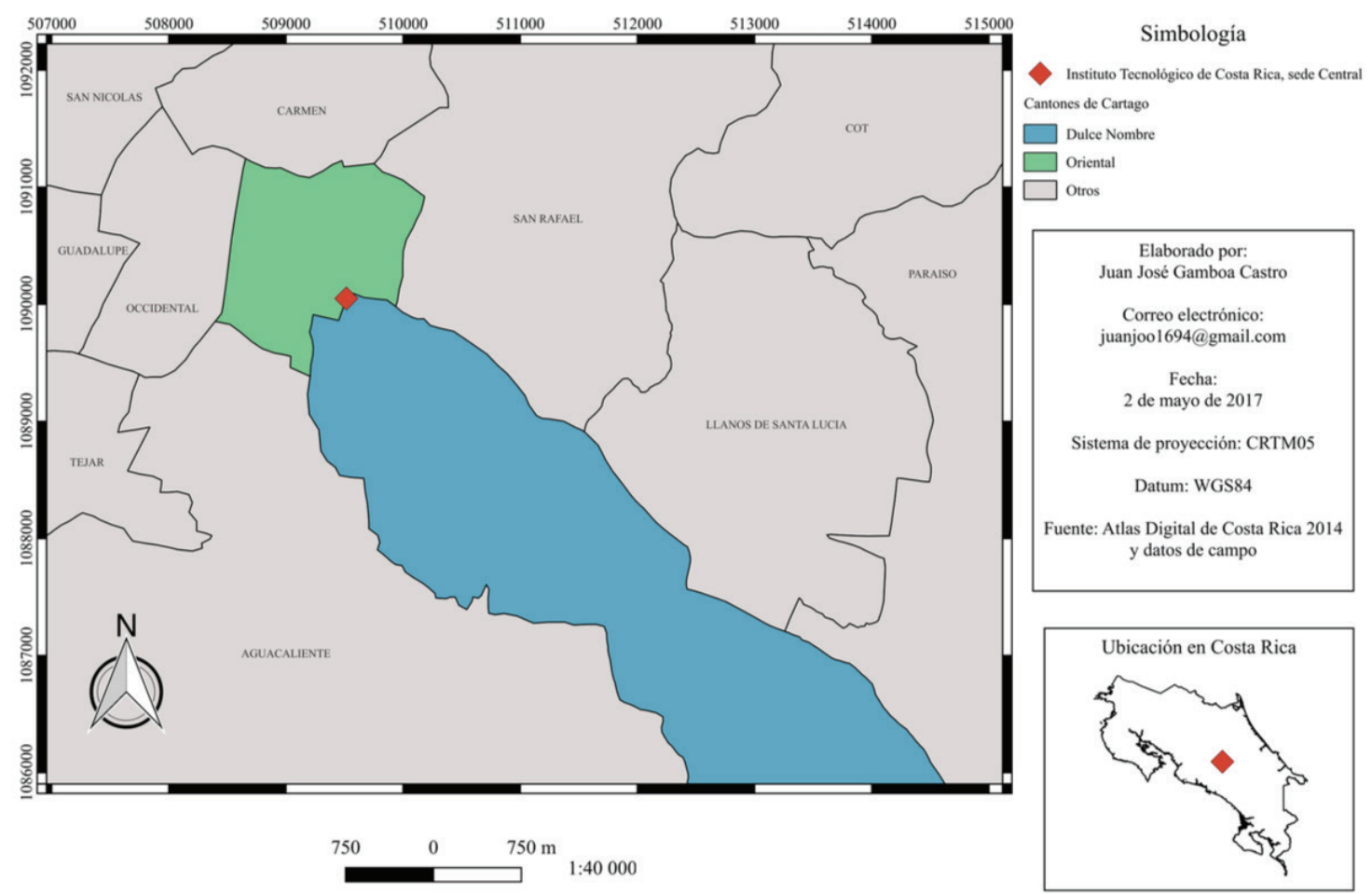

Figura 1 Ubicación del Tecnológico de Costa Rica, sede Central.

EI TEC es una Institución autónoma de educación superior universitaria, dedicada a la docencia, investigación y extensión de la tecnología y ciencias conexas [11]. En los últimos años su desempeño ambiental ha sido sobresaliente, obteniendo la más alta calificación otorgada por MINAE a su Programa de Gestión Ambiental Institucional (PGAI), además ha sido galardonada con Bandera Azul Ecológica en las categorías Centros Educativos y Cambio Climático para varios de sus recintos a nivel nacional. 
Con motivo de mantener sus altos estándares en materia de gestión ambiental y operar bajo el principio de mejora continua, el TEC se interesó en profundizar en la temática de CPV; para lo cual se contó con el apoyo de la Vicerrectoría de Administración, la Unidad Institucional de Gestión Ambiental y Seguridad Laboral y la Unidad de Proveeduría del Departamento de Aprovisionamiento.

\section{Priorización y selección de categorías de productos}

Con la finalidad de delimitar las categorías de productos (grandes grupos de bienes y servicios cuya naturaleza es semejante) para la realización de esta investigación, como primer filtro, se tomó en cuenta únicamente las categorías contenidas en la Guía de CPS (Dirección General de Administración de Bienes y Contratación Administrativa (DGABCA). [12], pues son en las que existe mayor experiencia.

En dicha guía, se encuentran priorizadas las 10 categorías de productos de mayor demanda en las instituciones del Estado costarricense [12], sin embargo, al no ser tan satisfactoria su implementación [10], es factible reforzar la inclusión de criterios ambientales desde una perspectiva de enfoque de ciclo de vida, analizando la realidad de los proveedores o posibles oferentes del TEC.

El alcance de los grupos de productos a incluir se consensuó con la Regente Ambiental de la Unidad Institucional de Gestión Ambiental y Seguridad Laboral del TEC y el coordinador de la Unidad de Proveeduría del Departamento de Aprovisionamiento; tomando en cuenta el estado actual de la Institución en la implementación de CPV, por lo que como segundo filtro, se analizaron un conjunto de pliegos de condiciones de contratos adjudicados y/o finiquitados relativos las categorías mencionadas, con el fin de determinar existencia o no de especificaciones técnicas ambientales en los criterios de admisibilidad o evaluación.

Finalmente, como tercer filtro, se adaptó la herramienta matriz de selección y priorización de bienes y servicios [5]. La adaptación consiste en una hoja de cálculo en Microsoft Excel Versión 15.31, en la que se sometieron las categorías de producto a una valoración frente a variables de selección y priorización como relación de gasto de objeto - presupuesto total, volumen de compra, impacto ambiental, entre otras; cada variable cuenta con una ponderación de acuerdo con su relevancia, así, los productos con los mayores valores numéricos fueron los prioritarios para la inclusión de criterios ambientales.

La ponderación de cada variable, así como sus grados, se definen en el cuadro 1.

\section{Criterios Ambientales y evidencias de verificación}

\section{Revisión de literatura}

Para la identificaron de criterios ambientales con su respectiva evidencia de verificación aplicables a las categorías de productos priorizadas, se realizó una revisión bibliográfica orientada a fuentes de países líderes en materia de CPV y CPS como los miembros de la UE, Japón, Estados Unidos y algunos casos en la región latinoamericana, específicamente Colombia, Chile y Costa Rica. La selección de estos criterios se basó en la frecuencia de aparición en las referencias bibliográficas y en su regulación por legislación nacional o normas voluntarias. 
Cuadro 1. Descripción de las variables de evaluación y escala para la matriz de selección y priorización de bienes y servicios en el ITCR. (Adaptado de [13]; [5]).

\begin{tabular}{|c|c|c|c|c|c|}
\hline Variable & Definición & $\begin{array}{c}\text { Ponderación } \\
(\%)\end{array}$ & Grado & Significado & Escala \\
\hline \multirow{3}{*}{$\begin{array}{l}\text { Relación de gasto de } \\
\text { objeto - presupuesto } \\
\text { total }\end{array}$} & \multirow{3}{*}{$\begin{array}{l}\text { Proporción (división } \\
\text { matemática) en } \\
\text { colones destinados } \\
\text { a la contratación del } \\
\text { bien o servicio sobre } \\
\text { el total destinado } \\
\text { a la subpartida del } \\
\text { presupuesto anual a } \\
\text { la que pertenece ese } \\
\text { objeto de gasto }\end{array}$} & \multirow{3}{*}{20} & 1 & Bajo & Relación < 3\% \\
\hline & & & 2 & Medio & $\begin{array}{c}\text { Relación entre } 4 \% \\
\text { y } 10 \%\end{array}$ \\
\hline & & & 3 & Alto & Relación > 11\% \\
\hline \multirow{3}{*}{ Volumen de compra } & \multirow{3}{*}{$\begin{array}{l}\text { Estimación cualitativa } \\
\text { de la cantidad del } \\
\text { producto que se } \\
\text { adquiere durante un } \\
\text { período de tiempo } \\
\text { determinado }\end{array}$} & \multirow{3}{*}{20} & 1 & Bajo & $\begin{array}{l}\text { Bajo volumen de } \\
\text { compra }\end{array}$ \\
\hline & & & 2 & Medio & $\begin{array}{c}\text { Volumen de } \\
\text { compra moderado }\end{array}$ \\
\hline & & & 3 & Alto & $\begin{array}{l}\text { Alto volumen de } \\
\text { compra }\end{array}$ \\
\hline \multirow{3}{*}{ Impacto ambiental } & \multirow{3}{*}{$\begin{array}{c}\text { Cantidad de aspectos } \\
\text { ambientales asociados } \\
\text { al producto bajo } \\
\text { la perspectiva de } \\
\text { enfoque de ciclo de } \\
\text { vida }\end{array}$} & \multirow{3}{*}{30} & 1 & Bajo & $\begin{array}{c}\text { El objeto de } \\
\text { gasto presenta } \\
\text { de } 1 \text { a } 4 \text { aspectos } \\
\text { ambientales }\end{array}$ \\
\hline & & & 2 & Medio & $\begin{array}{c}\text { El objeto de } \\
\text { gasto presenta } \\
\text { de } 5 \text { a } 8 \text { aspectos } \\
\text { ambientales }\end{array}$ \\
\hline & & & 3 & Alto & $\begin{array}{c}\text { El objeto de } \\
\text { gasto presenta } 9 \\
\text { O más aspectos } \\
\text { ambientales }\end{array}$ \\
\hline \multirow{3}{*}{$\begin{array}{c}\text { Cobertura interna de } \\
\text { uso }\end{array}$} & \multirow{3}{*}{$\begin{array}{l}\text { Estimación cualitativa } \\
\text { del bien o servicio } \\
\text { que se utiliza por los } \\
\text { funcionarios de la } \\
\text { Institución }\end{array}$} & \multirow{3}{*}{30} & 1 & Bajo & $\begin{array}{l}\text { Bajo nivel de uso/ } \\
\text { consumo }\end{array}$ \\
\hline & & & 2 & Medio & $\begin{array}{c}\text { Moderado nivel de } \\
\text { uso/consumo }\end{array}$ \\
\hline & & & 3 & Alto & $\begin{array}{l}\text { Alto nivel de uso/ } \\
\text { consumo }\end{array}$ \\
\hline
\end{tabular}

\section{Entrevista a proveedores de las categorías de productos}

Uno de los factores medulares en la implementación de las CPV es fiscalizar el cumplimiento de las especificaciones técnicas ambientales que se solicitan en el pliego de condiciones, es por ello que, se construyó una entrevista semiestructurada [14] para proveedores de cada uno de los sectores que se abarcan en esta investigación, la finalidad las mismas fue determinar la factibilidad de las empresas para ofrecer bienes y servicios con menor impacto ambiental.

A continuación, se presenta la estructura de dichas entrevistas:

- Objetivo.

- Información general sobre el entrevistado y la empresa.

- Consentimiento informado.

- Clasificación de la empresa de acuerdo con su tamaño (grande, mediana, pequeña o micro). 
- Cuadros que incluyen los criterios ambientales clasificados según la etapa del ciclo de vida del producto, opciones de respuesta (sí, no, desconoce, no aplica) para determinar si la empresa satisface el criterio ambiental, una columna denominada "Evidencia" donde se recopiló el mecanismo de verificación que utiliza la empresa, , una columna con las evidencias de verificación según lo recopilado en la revisión bibliográfica y una fila denominada "Otros" con la finalidad de que la empresa propusiera criterios ambientales con sus respectivas evidencias..

- Tres preguntas abiertas acerca de barreras y beneficios de la inclusión de criterios ambientales dentro de los bienes o servicios que ofrecen al mercado, consultas dirigidas únicamente a aquellas empresas que consideran sus productos como "verdes".

La base para la elaboración de las entrevistas fue la revisión bibliográfica de criterios ambientales y evidencias de verificación. Al provenir la mayoría de esta información de fuentes internacionales, los criterios se adecuaron a la realidad costarricense.

\section{Selección de empresas a entrevistar}

Se siguió un muestreo cualitativo no probabilístico por cuotas, cuya finalidad fue seleccionar casos o unidades que ayudaran a comprender detalles y actores del fenómeno en estudio, sin pretender hacer una generalización a la población en términos de probabilidad [14]. De acuerdo con Hernández et al. [14], el tamaño mínimo de la muestra para estudios de teoría fundamentada o entrevistas es de 20 a 30 casos, para esta investigación se propuso en 20, teniéndose 5 empresas por cada categoría de producto. La elección de las empresas depende de las características de la investigación [15], por ejemplo, para este caso en particular se tomó en cuenta dos clases de proveedores: los que resultan adjudicados con mayor frecuencia en el TEC [16], y la segunda clase, empresas que no necesariamente hayan sido proveedores de la Institución pero que afirmen ofrecer productos con características ambientales o sostenibles, para ello se contactó con la Asociación Empresarial para el Desarrollo y la Cámara de Industrias de Costa Rica, también se visitó el portal web Páginas Verdes de Costa Rica [17].

\section{Análisis de entrevistas}

A partir de los hallazgos obtenidos en las entrevistas, se realizó el cálculo de la frecuencia relativa para cada criterio para las clases enunciadas en la investigación realizada por GamboaCastro y Salazar-Rojas [17].

\section{Clasificación de criterios ambientales}

En concordancia con el Decreto N ${ }^{\circ} 33411-H$ Reglamento a la Ley de Contratación Administrativa [18] y la NTACS [12], se clasificaron los criterios ambientales recopilados como admisibilidad o evaluación. Los criterios de admisibilidad son aquellos requisitos mínimos estipulados en el pliego de condiciones que deben cumplir los posibles oferentes, sin los cuales no podrían avanzar a las siguientes etapas del proceso licitatorio. Por su parte, los criterios de sistema de evaluación corresponden a requisitos deseables mas no indispensables para considerarse elegible y que se determina su cumplimiento después de haber superado la etapa de acatamiento de criterios de admisibilidad [18]. En esta investigación, para clasificar los criterios recopilados como admisibilidad o evaluación, se revisó la normativa aplicable a las categorías de productos en el Sistema Costarricense de Información Jurídica (SCIJ) de la Procuraduría General de la República (PGR), en el Sistema de Reglamentación Técnica del MEIC y en el catálogo de normas técnicas del Instituto de Normas Técnicas de Costa Rica (INTECO), de modo que, si existe legislación que avale el criterio ambiental, su clasificación es de admisibilidad; de lo contrario, se recurrió a los resultados de las entrevistas a los proveedores y se determinó 
en conjunto con la Regente Ambiental del TEC una tasa mínima de cumplimiento del criterio con evidencia objetivamente verificable, para clasificarlo como admisibilidad, en caso de que el porcentaje obtenido sea menor, se clasificó como evaluación.

\section{Limitaciones}

Las empresas entrevistadas fueron seleccionadas por haber sido proveedoras de la Institución o por ofrecer productos que incluyen la variable ambiental dentro de su ciclo de vida; no se pretende generar inferencias estadísticas significativas a la situación de Costa Rica. El análisis efectuado es particular para el TEC, lo cual podría aproximarse a la realidad de otras instituciones públicas, sin embargo, no es objeto de esta investigación comprobar si existe tal correlación.

Asimismo, se asume la veracidad de las respuestas recolectadas en las entrevistas. Por último, el factor económico (costo de los bienes y servicios) se excluyó del estudio, al ser dinámico y dependiente de múltiples elementos como la cantidad de bienes a adquirir, la capacidad de negociación de la Institución con los proveedores, la situación del mercado, entre otros.

\section{Resultados y discusión}

\section{Priorización y selección de categorías de productos}

Inicialmente se aplicó el primer filtro para seleccionar las categorías de bienes y servicios, considerando solo aquellas incluidas en la Guía de CPS del Ministerio de Hacienda [12]: equipo de cómputo, equipo de oficina, impresión y reproducción, limpieza, suministros de oficina, vehículos, servicios de alimentación, vestuario, publicidad y servicios de vigilancia; esto debido a que dichos grupos representan las áreas de gasto en las que existe experiencia a nivel nacional e internacional, guías y conocimiento para implementar CPV, en general, son los de mayor demanda en el sector público [12], [2], [16]. La categoría de servicios de vigilancia fue excluida, ya que en el caso del TEC, estos servicios no se contratan, al contar con funcionarios permanentes para ello.

En la figura 2 se presenta el presupuesto destinado en el 2016 para los objetos de gasto incluidos en el primer filtro.

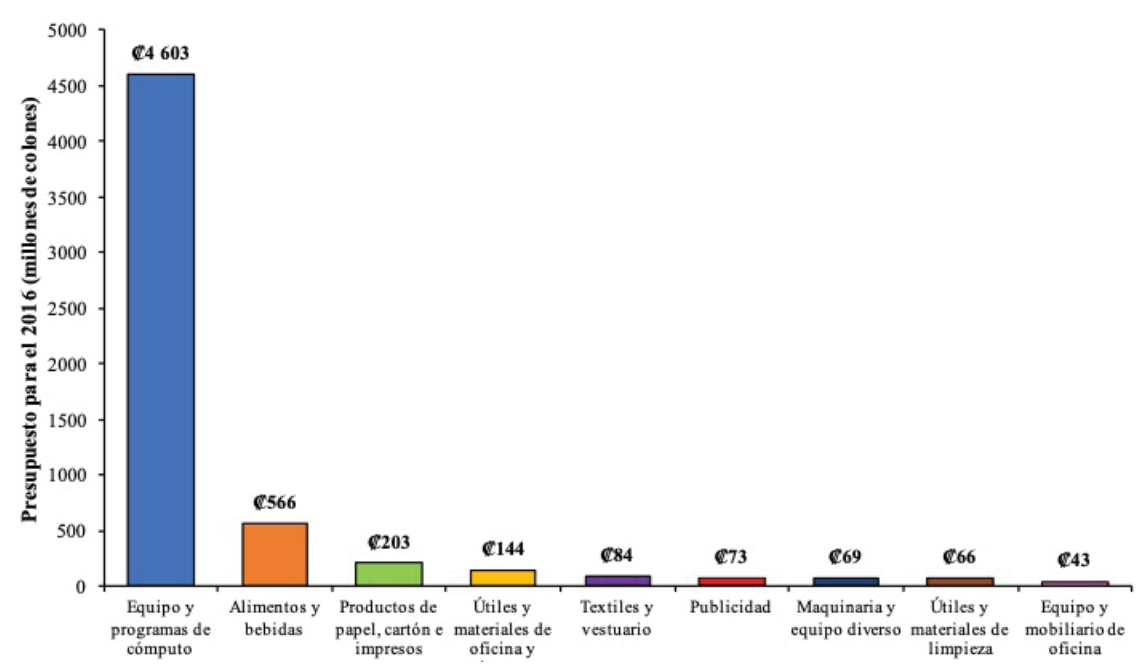

Figura 2. Presupuesto 2016 consignado a las nueve categorías de productos aplicables al TEC de la Guía de CPS de Costa Rica. 
De acuerdo con la figura 2, la categoría de productos con mayores recursos para su contratación de los nueve incluidos en el primer filtro fue equipo y programas de cómputo, lo cual tiene sentido al ser el TEC una institución de educación superior predominantemente de ingenierías, por lo que su inversión en este rubro es significativa; seguido por alimentos y bebidas destinados a los Restaurantes Institucionales y servicios de alimentación temporales para eventos y actos protocolarios, posteriormente productos de papel, cartón y otros, el cual es relativamente bajo debido a los esfuerzos que hace la Institución en la digitalización de servicios y procesos; el siguiente objeto en orden de gasto son los útiles y materiales de oficina, seguido de textiles y vestuario, publicidad, maquinaria y equipo diverso (donde se incluyen los vehículos), implementos de limpieza, por último, mobiliario de oficina.

Como segundo filtro, se realizó el análisis de los pliegos de condiciones más recientes acerca de estos nueve objetos de gasto, se evidenció que la adquisición de equipo de cómputo y suministros de limpieza actualmente cuentan con especificaciones técnicas ambientales dentro del sistema de evaluación, es decir, se otorgan puntos adicionales a los concursantes que presenten evidencias referente a estos criterios, por este motivo, se excluyeron del alcance de este estudio, para incursionar en otras áreas donde exista total carencia de los mismos, dichas áreas son las restantes: equipo de oficina, impresión y reproducción, suministros de oficina, vehículos (maquinaria y equipo diverso), servicios de alimentación y vestuario.

Como última etapa para la determinación de las categorías de productos, se aplicó la adaptación de la matriz de selección y priorización de bienes y servicios obteniéndose en el cuadro 2 los resultados obtenidos.

Con base en los resultados anteriores, se seleccionaron las siguientes categorías de productos: mobiliario y suministros de oficina, servicios de alimentación y vestuario, categorías semejantes a las obtenidas por M. Alonso [19] y B. Pacheco \& M. Bastante [20] en estudios similares. A continuación, se define el alcance de cada una de estas categorías:

- Mobiliario de oficina: productos destinados al soporte y organización de implementos de oficina como escritorios, estantes, armarios.

- Suministros de oficina:

Papel de impresión y escritura: incluye el papel utilizado en oficinas para impresión, fotocopias, escritura y usos cotidianos.

Suministros varios: material de archivo (carpetas y archivadores), fundas plásticas, derivados del papel (libretas, blocs de notas, sobres manila y de correspondencia, notas autoadhesivas), bolígrafos, marcadores permanentes, acrílicos (para pizarra) y resaltadores, corrector líquido, lápices de grafito, cintas adhesivas y pegamento, tijeras, grapadoras, sacapuntas y gomas de borrar.

- Vestuario: incluye uniformes de seguridad, camisetas y textiles en general.

- Servicios de alimentación:

Temporales (catering service): servicios contratados por la Institución para eventos ocasionales y actos protocolarios.

Restaurante Institucional: servicio permanente administrado por el TEC, esta subcategoría se limitó a la inclusión de criterios ambientales para frutas, verduras, hortalizas y carne (pescado, res y pollo). 
Sodas concesionadas: locales destinados a proveer servicios de alimentación dentro del TEC, cuya administración no es competencia directa de la Institución. Para esta subcategoría, el alcance incluye únicamente criterios ambientales de ejecución del servicio.

Cuadro 2. Resultados de la matriz de selección y priorización de bienes y servicios para la implementación de CPV en el TEC.

\begin{tabular}{|c|c|c|c|c|c|}
\hline $\begin{array}{c}\text { Bienes y } \\
\text { servicios }\end{array}$ & $\begin{array}{c}\text { Relación } \\
\text { de gasto/ } \\
\text { presupuesto anual } \\
(20 \%)\end{array}$ & $\begin{array}{c}\text { Volumen } \\
\text { de compra } \\
(20 \%)\end{array}$ & $\begin{array}{c}\text { Impacto } \\
\text { Ambiental } \\
(30 \%)\end{array}$ & $\begin{array}{c}\text { Cobertura } \\
\text { interna de uso } \\
(30 \%)\end{array}$ & Ponderación \\
\hline $\begin{array}{c}\text { Mobiliario de } \\
\text { oficina }\end{array}$ & 1 & 3 & 2 & 3 & 2,3 \\
\hline $\begin{array}{c}\text { Impresión y } \\
\text { reproducción }\end{array}$ & 3 & 1 & 1 & 3 & 1,7 \\
\hline $\begin{array}{c}\text { Suministros de } \\
\text { oficina }\end{array}$ & 3 & 1 & 3 & 3 & 3 \\
\hline Vehículos & 1 & 3 & 3 & 3 & 3 \\
\hline $\begin{array}{c}\text { Servicios de } \\
\text { alimentación }\end{array}$ & 3 & 2 & 3 & 2 & 2 \\
\hline Vestuario & 2 & & & \\
\hline
\end{tabular}

Criterios ambientales y evidencias de verificación

Utilizando el enfoque cualitativo de ciclo de vida, se determinaron los criterios ambientales con su respectiva evidencia de verificación para las 4 categorías de productos en estudio. En general, los criterios están orientados al uso de materiales, reciclados, reciclables y menos impactantes al ambiente, disminución de generación de residuos, facilidad de reparación, procedencia de materias primas, menos uso de material de empaque, entre otros [6]. Además de los beneficios en materia de sostenibilidad de los criterios ambientales al momento de planificar y realizar una compra, la Institución contratante asegura la trazabilidad en el proceso productivo, esto brinda la certeza de que se realizó una compra responsable y que dio correcto uso a la Hacienda Pública.

\section{Entrevista a proveedores de las categorías de productos}

\section{Caracterización de la muestra analizada}

Se entrevistaron 30 empresas que comercializan las categorías de productos analizadas en este estudio. La distribución de acuerdo con su tamaño se muestra en la figura 3, la mayoría de los proveedores consultados corresponden a microempresas (43\%), seguido de medianas (23\%), por último, grandes y pequeñas (17\% para cada grupo). Lo anterior implica que para este estudio las PYMES tuvieron un papel primordial, reflejo de la realidad nacional, pues de acuerdo con datos del Ministerio de Economía, Industria y Comercio [21] el parque empresarial de Costa Rica está conformado por un 93,4\% de PYMES y el restante 6,6\% corresponde a grandes empresas. 
En cuanto a la distribución de entrevistados de acuerdo con la categoría de producto, la figura 4 muestra que un 30\% se encarga del comercio de productos textiles y la misma tasa se dedica a servicios de alimentación. Por su parte, un 23\% distribuyen suministros de oficina, y la menor participación, con un 17\%, es del sector de confección y venta de mobiliario de oficina, esto como consecuencia de que no se localizaron empresas que se publiciten como verdes, por tanto, se entrevistó únicamente a las que han sido proveedoras institucionales.

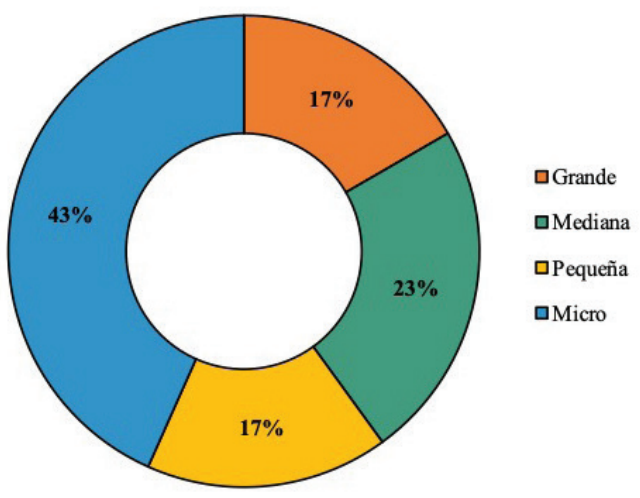

Figura 3. Distribución porcentual respecto a la clasificación de las empresas entrevistadas de acuerdo con su tamaño.

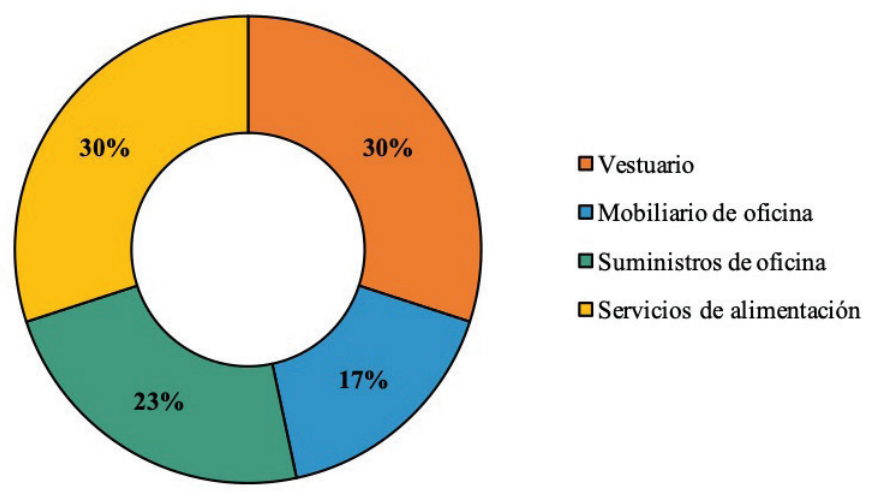

Figura 4. Distribución porcentual respecto a la actividad comercial de las empresas entrevistadas.

Análisis general de cumplimiento de criterios ambientales

En la figura 5 se presentan los resultados globales obtenidos en las entrevistas para cada categoría de producto. A partir de esta se concluye que los productos con mayor porcentaje de cumplimiento de los criterios con evidencia objetivamente verificable son la categoría de mobiliario de oficina (54\%) y la subcategoría de papel para impresión y escritura (53\%), ambos tipos de artículos derivados de la madera, para la cual existe mayor capacidad de trazabilidad y consideraciones ambientales a lo largo de su ciclo de vida por medio de certificaciones ampliamente extendidas a nivel mundial. Mientras que las subcategorías de Restaurante Institucional (32\%) y suministros varios (39\%) presentan las menores tasas de satisfacción de las especificaciones técnicas ambientales consultadas.

A su vez, de la categoría de mobiliario de oficina (31\%) y la subcategoría de papel para impresión y escritura (53\%) se obtuvo los mayores niveles de incumplimiento de criterios, específicamente en etapas del ciclo de vida como manufactura y embalaje. Por su parte, el más alto desconocimiento de los criterios ambientales y su acatamiento recae en el vestuario 
(100\%) y en los suministros varios (37\%), situación que ocurre como consecuencia de que los entrevistados cumplen la función de distribuidores, es decir, no tienen control sobre la selección de materias primas y el proceso productivo.

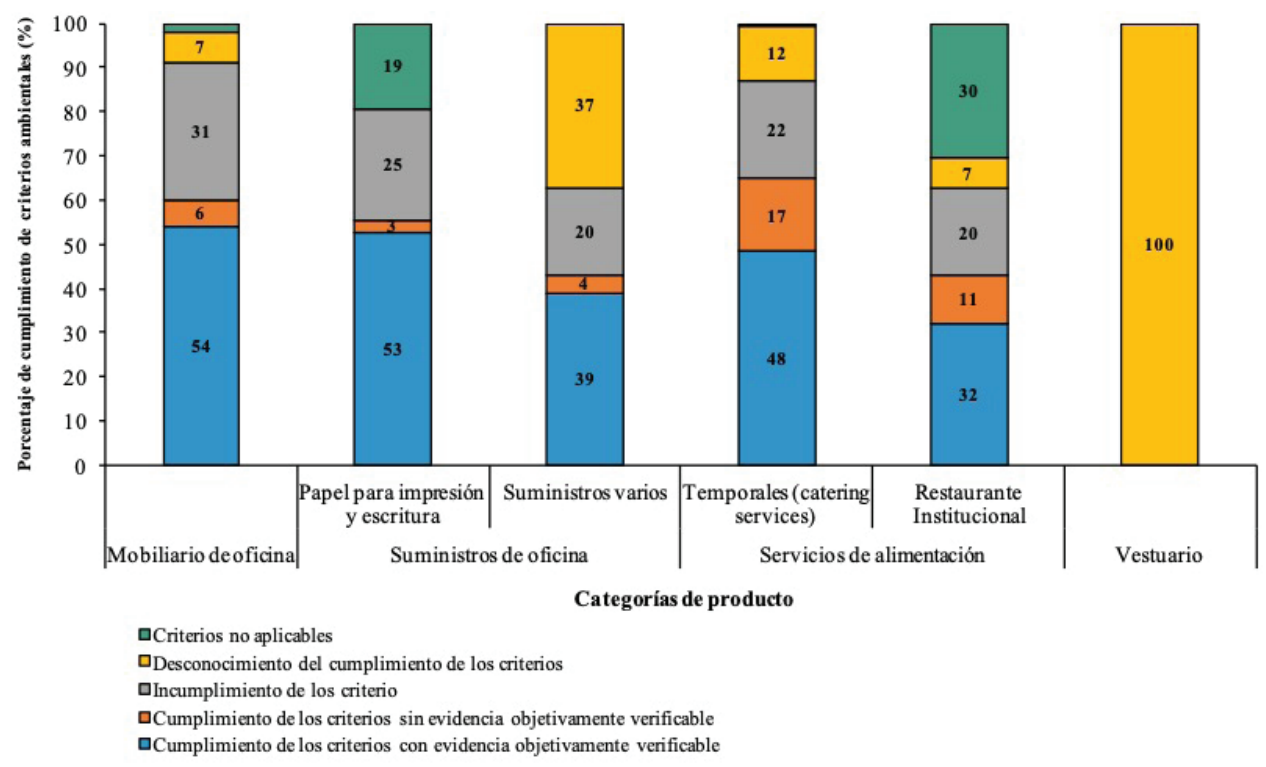

Figura 5. Distribución porcentual del cumplimiento de criterios ambientales para las categorías de productos.

\section{Clasificación de criterios ambientales}

Según la NTACS [12] entre otras consideraciones, un criterio de admisibilidad debe hacer referencia a experiencia mínima del proveedor, aspectos básicos de capacidad financiera, características técnicas indispensables y cumplimiento básico de legislación que incluya explícitamente el criterio; de la lista anteriormente mencionada, se seleccionó la última condición para realizar la clasificación, pues el cumplimiento legal es objetivamente verificable para justificar la inclusión de una especificación técnica ambiental dentro de los requisitos indispensables de un pliego de condiciones, además de que no debe otorgarse puntación adicional por su cumplimiento.

Por otra parte, esta misma normativa [12] faculta a las instituciones a realizar un estudio preliminar de mercado, con el fin de esclarecer la sección del cartel en la cual se deben asignar los criterios, y evitar lo que implica la resolución infructuosa de un proceso de contratación administrativa, es por ello que, con base en las entrevistas realizadas se definió en consenso con la Regente Ambiental del TEC que, si el criterio ambiental presenta una tasa de cumplimiento con evidencia objetivamente verificable mayor al 50\%, se cataloga de admisibilidad y menor a este límite, se clasifica como de evaluación.

Para los criterios que solo es posible verificarlos durante la ejecución del contrato, pese a estar clasificados como admisibilidad o evaluación (criterios de seguimiento a contrato), es necesario agregar una cláusula de sanciones por incumplimiento durante la ejecución del contrato, tal como lo estipula la NTACS [12].

En la figura 6 se presenta el resultado de la clasificación de los criterios ambientales para cada una de las categorías, inclusive los sugeridos por los proveedores. Esto permite una visualización general, de que, para las categorías estudiadas a excepción de servicios de alimentación temporal, la mayoría de los criterios se clasifican como de evaluación al no ser 
un requisito legal o ser satisfecho por el 50\% o más de los proveedores consultados. Lo cual evidencia una falta de madurez en el tema a nivel de legislación y en las formas de producción y distribución de productos y servicios por parte de los proveedores.

Para la categoría de mobiliario de oficina, se obtuvo un $44 \%$ de admisibilidad con un $12 \%$ que cuentan con algún tipo de normativa que respalde su inclusión dentro de los requisitos de admisibilidad y un $32 \%$ de los criterios resultaron de admisibilidad según el parámetro definido y $56 \%$ de evaluación. Los criterios para el sistema de evaluación son aquellos que implican innovación en el diseño, uso de materiales novedosos y retan la creatividad del proveedor.

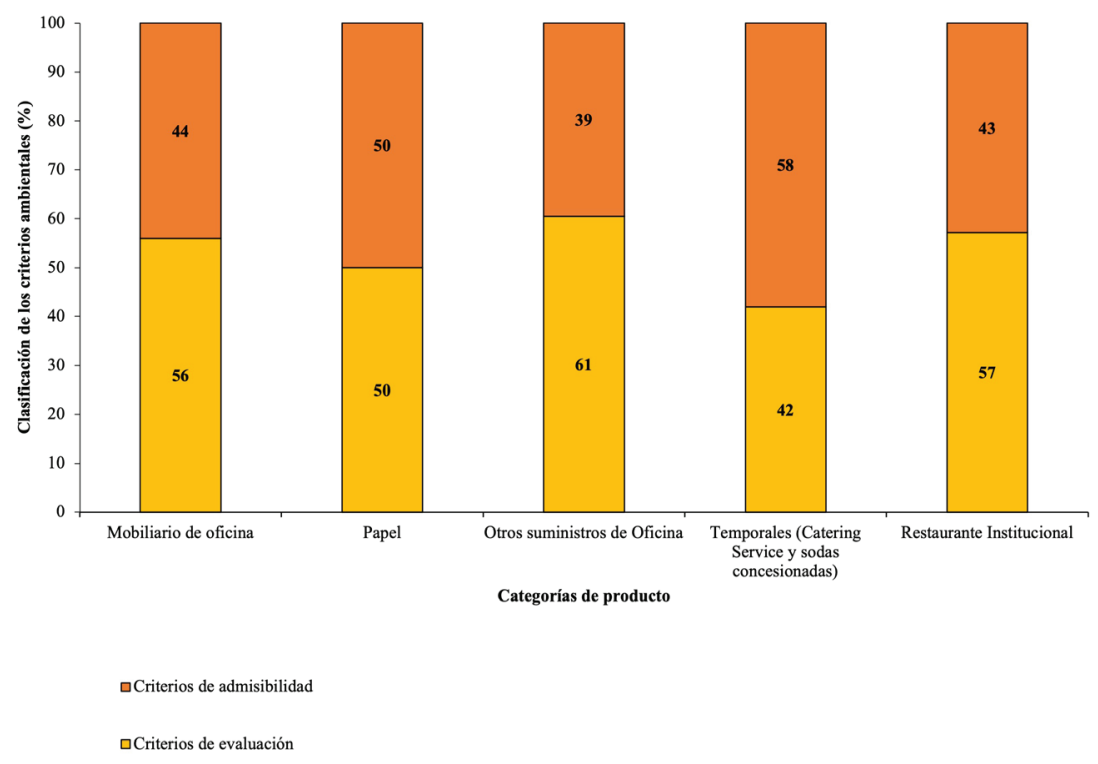

Figura 6. Distribución porcentual de la clasificación de los criterios ambientales según las categorías de productos.

De acuerdo a la figura 6, para los servicios de alimentación temporales, el 58\% de los criterios evaluados fueron de admisibilidad 35\% cuentan con normativa jurídica y voluntaria aplicable, un porcentaje significativo respecto a otras categorías, esto debido a que los productos comestibles como cárnicos y agrícolas cuentan con mayor regulación por su importancia en la salud pública y un $42 \%$ de las especificaciones se catalogaron como de evaluación, puesto que criterios donde se solicitan certificaciones voluntarias como la de INCOPESCA, MarViva y SENASA, entre otras, no se encuentran difundidos entre los proveedores entrevistados como consecuencia del insipiente avance de las mismas a nivel nacional. Lo mismo ocurrió con la subcategoría de Restaurante Institucional donde se obtuvo un $43 \%$ de criterios para admisibilidad (21\% con normativa) y un 57\% para evaluación.

Para la categoría de Servicios de alimentación, se dificulta un poco más la inclusión de criterios ambientales, ya que la mayoría de los proveedores cumplen la función de distribuidores, es decir, no tienen control sobre las materias primas ni procesos de manufactura, por ello, deben ir hacia atrás en la cadena de suministro para exigir las prácticas ambientales que se solicitan en los carteles. La clasificación de los criterios irá cambiando paulatinamente hasta que el mercado sea capaz de ofrecer los de evaluación como admisibilidad.

Con respecto al vestuario la normativa relacionada a textiles corresponde al Reglamento Técnico Centroamericano. Textiles y productos textiles. Requisitos de etiquetado. RTCA 51.01.08:12 y el Decreto No 25989-MEIC RTCR 235:1997. Productos textiles. Definiciones y requisitos; no obstante, ninguna de estas dos incluye criterios ambientales para prendas y afines, debido a 
esto y a los resultados obtenidos en las entrevistas, podrán incluirse en el sistema de evaluación del pliego de condiciones, hasta que la industria textil costarricense demuestre avances en materia de sostenibilidad.

\section{Resumen de Propuesta de Metodología para Compras con Criterios Ambientales}

En la figura 7 se presenta un diagrama de flujo de la metodología propuesta, en este caso, aplicada al TEC.

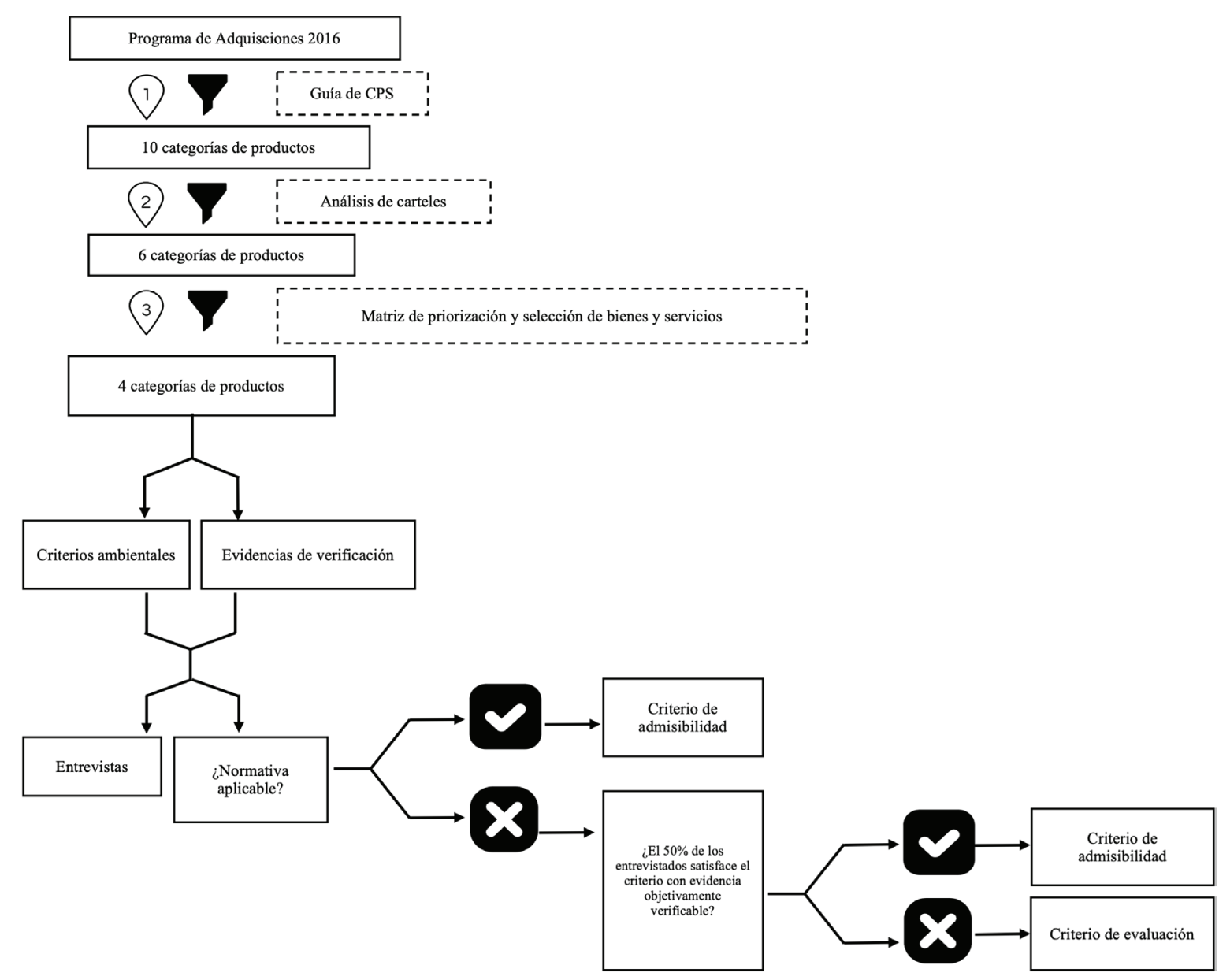

Figura 7. Diagrama de flujo de la metodología propuesta para el estudio de caso en el TEC.

De esta manera la metodología propuesta para la inclusión de criterios ambientales en las compras públicas de Costa Rica requiere la priorización de categorías de productos, definición de especificaciones técnicas ambientales, evaluar la factibilidad de los proveedores para cumplir los criterios, esto considerando que aún no se tienen la madurez de mercado con la suficiente oferta y solicitar algo que el mercado no ofrece llevaría a procesos infructuosos que a la larga implican mayores gastos administrativos al tener que volverse a realizar, y la adecuada clasificación de estos dentro del pliego cartelario para evitar procesos de contratación administrativa infructuosos. Un ejemplo de aplicación de esta metodología, conteniendo los criterios ambientales y su respectivo análisis para la categoría de suministros de oficina puede ser encontrado en el estudio realizado por Gamboa-Castro y Salazar-Rojas [17]. 


\section{Conclusiones}

- De las categorías de productos priorizadas, mobiliario, suministros de oficina, vestuario y servicios de alimentación, la que obtuvo mayor porcentaje de cumplimiento de las especificaciones técnicas ambientales con evidencia objetivamente verificable fueron mobiliario de oficina (54\%) y la subcategoría de papel para impresión y escritura (53\%), debido a que existe mayor capacidad de trazabilidad por medio de certificaciones ampliamente extendidas a nivel mundial.

- Se obtuvo una mayor cantidad de criterios clasificados como de evaluación, esto debido se clasificó un $11 \%$ de los criterios evaluados como admisibilidad por cumplimiento del primer criterio, y por el segundo criterio un 58\% de la totalidad de criterios evaluados se clasificaron como de evaluación y el restante 31\% fueron de admisibilidad al ser cumplidos con evidencia objetivamente verificable.

- Se evidencia la falta de madurez en términos ambientales de la muestra de empresas de la investigación, la mayoría no cuenta con información concreta sobre los aspectos ambientales de los productos que ofrecen al mercado, esta deficiencia fue extrema para el caso de los textiles.

- El análisis de los servicios de alimentación evidenció la falta de capacitación de las empresas y el insuficiente avance de programas de eco etiquetado en productos cárnicos a nivel nacional.

\section{Referencias}

[1] Organización de las Naciones Unidas (ONU). (2015). Transformar nuestro mundo: la Agenda 2030 para el Desarrollo Sostenible [Online]. Available: http://www.un.org/ga/search/view_doc.asp?symbol=A/ RES/70/1\&referer=/english/\&Lang=S

[2] Comisión de las Comunidades Europeas. (2008). Public procurement for a better environment [Online]. Available: http://eur-lex.europa.eu/legal-content/EN/TXT/PDF/?uri=CELEX:52008DC0400\&from=EN

[3] S. Brammer and H. Walker, "Sustainable procurement in the public sector: an international comparative study", International Journal of Operations e Production Management, vol. 31, no. 4, pp. 452-476, 2011.

[4] International Institute for Sustainable Development (IISD). (2015). Handbook for the Inter-american Network on Government Procurement (INGP): Implementing Sustainable Public Procurement in Latin America and the Caribbean [Online]. Available: http://www.iisd.org/library/handbook-ingp-public-procurers-manual-para-agentes-de-compras-publicas-de-las-ricg\%0D

[5] Centro Nacional de Producción Más Limpia de Colombia. (2013). Guía Conceptual y Metodológica de Compras Públicas Sostenibles [Online]. Available: http://www.minambiente.gov.co/images/ AsuntosambientalesySectorialyUrbana/pdf/compras públicas/guia compras publicas sostenibles.pdf

[6] Manual para la implementación de Compras Verdes en el sector público de Costa Rica, Fundación Centro de Gestión Tecnológica e Informática Industrial (CEGESTI), San José, Costa Rica, 2008.

[7] Manual Práctico de Compra y Contratación Pública Verde. Ihobe Sociedad Pública de Gestión Ambiental del Gobierno Vasco, España, 2014.

[8] Centro Nacional de Producción Más Limpia de Colombia. (2013). MEDIOS DE VERIFICACIÓN DE LOS CRITERIOS DE SOSTENIBILIDAD [Online]. Available: https://www.minambiente.gov.co/images/ AsuntosambientalesySectorialyUrbana/pdf/compras públicas/guia compras publicas sostenibles.pdf

[9] Guía para la elaboración de Programas de Gestión Ambiental Institucional (PGAl) en el sector público de Costa Rica, Ministerio de Ambiente y Energía (MINAE), San José, Costa Rica: Ministerio de Ambiente y Energía (MINAE).

[10] Fundación Centro de Gestión Tecnológica e Informática Industrial (CEGESTI). (2013) Estado de implementación de las compras públicas sustentables en Costa Rica [Online]. Available: http://www.hacienda.go.cr/ docs/56e060bd56a07_Estado implementación compras sustentables Costa Rica.pdf

[11] Instituto Tecnológico de Costa Rica (TEC). (2015). Acerca del TEC [Online]. Available: http://www.tec.ac.cr/ eltec/Paginas/acercaDelTec.aspx 
[12] Guía de Compras Públicas Sustentables, Dirección General de Administración de Bienes y Contratación Administrativa (DGABCA), San José, Costa Rica, 2011.

[13] D. Acosta and M. Vanegas, "Compras Corporativas Sostenibles -Coca-Cola", Universidad Pontificia Bolivariana [Online], Available: https://repository.upb.edu.co/bitstream/handle/20.500.11912/2910/Compras Corporativas Sostenibles- Acosta-Vanegas. pdf?sequence=1\&isAllowed=y\%0D

[14] R. Hernández et al., "Recolección y análisis de los datos cualitativos" in Metodología de la investigación, 6th ed. México: McGraw-Hill, pp. 403-406

[15] C. Ragin, "The Comparative Method: Moving Beyond Qualitative and Quantitative Strategies". Univ. of California Press, CA, 2014.

[16] Programa de Naciones Unidas para el Medio Ambiente (PNUMA). (2012) Implementando Compras Públicas Sostenibles. Introducción al Enfoque de PNUMA [Online]. Available: http://www.unep.org/resourceefficiency/ Portals/24147/scp/10yfp/document/SPPguidelines SP 27.07.12.pdf\%0D

[17] J. Gamboa-Castro and T. Salazar-Rojas, "Inclusión de Criterios ambientales en las Compras Públicas en Costa Rica: Suministros de Oficina”. Tecnología en Marcha, vol. 31, no.4, pp. 167-185, 2011.

[18] Decreto Ejecutivo No 33411-H Reglamento a la Ley de Contratación Administrativa, Ministerio de Hacienda, 2006.

[19] Alonso, M. (2010). COMPRA PÚBLICA SOSTENIBLE -Un estudio comparativo entre los programas regionales para la compra publica sostenible, en España. Universidad de Ciencias Agrícolas de Suecia. Recuperado a partir de http://stud.epsilon.slu.se/1884/2/alonso_m_101005_ex_summary.pdf

[20] Pacheco, B., \& Bastante, M. "Green public procurement as an initiative for sustainable consumption. An exploratory study of Spanish public universities". Journal of Cleaner Production, vol. 133, pp. 648-656, 2016.

[21] Ministerio de Economía Industria y Comercio (MEIC). (2015) ESTADO DE SITUACIÓN DE LAS PYME EN COSTA RICA [Online]. Available: http://reventazon.meic. go.cr/informacion/estudios/2016/pymes/informe.pdf

[22] Decreto Ejecutivo № 25984-MEIC RTCR 282:1997. Artículos de oficina y escolares. Etiquetado de los productos de papelería y afines para uso escolar y de oficina, Ministerio de Economía Industria y Comercio, 1997. 